\title{
Effect of Body Mass Index and Gender on Visual and Auditory Reaction Times in Young Adults
}

\author{
Aiesha DurrebarYounus Khan, Amalladinna Ashwini and Basavaraj Malipatil \\ Department of Physiology, Mahadevappa Rampure Medical College, Rajiv Gandhi University of Medical Sciences, Kalaburagi \\ 585105, Karnataka, India
}

\begin{abstract}
AIM AND OBJECTIVE: Reaction time is defined as an interval of time between the application of stimulus and the initiation of appropriate voluntary response as the subject has been instructed to respond as rapidly as possible. Reaction time is found to be altered by a number of factors both physiological and pharmacological. Our aim was to study the effect of BMI (Body Mass Index) and gender on audiovisual reaction time. METHODS: The study was carried on 122 healthy medical students in the age group of 17-22 years, among them 68 were males and 54 were females. The subjects were presented with two visual stimuli (green and red light stimuli) and two auditory stimuli (tone and click sound stimuli). Data was analyzed using one-way ANOVA (analysis of variance). RESULTS: Individuals with a BMI less than, and individuals with a BMI greater than the normal range of BMI were found to have reaction times longer than those individuals having normal BMI. Therefore, in order to have the quickest reaction time, one needs to be healthy and fit and should have a BMI within the normal range. It was found that females had significantly longer visual reaction time for red light when compared to males. Even the auditory reaction time was also more in females than males, although it was not statistically significant.
\end{abstract}

Key words: gender, auditory reaction time, visual reaction time, body mass index.

\section{Introduction}

Reaction time can be defined as the time interval between the application of a stimulus and appearance of appropriate voluntary response by a subject [1]. For example, how fast would a person react to apply breaks of a vehicle during driving or how quick would a person flee from a site of danger. This topic is gaining much importance in the field of sports where accurate precise decisions and coordinated movements would change the whole game scenario. The reaction time can therefore be said to reflect the integrity and processing ability of the central nervous system and hence can be used as a measure of cognition [2].

Time and again, the discussions have cropped up regarding which gender has a faster reaction time. There are several studies indicating varied results where some have illustrated females having faster

Corresponding author: Aiesha DurrebarYounus Khan, MBBS, research field: human physiology. Email: durrebaryounus@yahoo.com processing abilities than their male counterparts while some studies have proved otherwise. Accounting these findings to the various physiological processes that alter the reaction time, the effect of BMI (Body Mass Index) has also been implicated to influence reaction time.

Considering the reaction time as a good indicator of sensorimotor coordination and performance of an individual; the present study was undertaken to study the effect of gender and BMI on the auditory reaction time and visual reaction time.

\section{Materials and Methods}

This cross sectional study was carried out in the Department of Physiology, Mahadevappa Rampure Medical College, India with prior approval from the Head of Department of Physiology.

\subsection{Study Group}

The study pattern and objectives were explained to 
medical undergraduate students. 122 students volunteered to be part of the study. The study group thus comprised of 122 apparently healthy medical students of the age group of 17-22 years amongst which 68 were males and 54 were females. Written informed consent was taken from those who volunteered.

Tests for hearing, vision and motor system examination including reflexes in upper limb were carried out on the subjects to rule out any auditory, visual and neuromuscular disorders, respectively.

\subsection{Recording of Anthropometrical Parameters}

Age and gender of each subject were noted. The height of the subjects was measured using a stadiometer whose least count was $1 \mathrm{~cm}$. The recording was then converted into unit of meters. Weight was measured using weighing machine whose least count was $0.5 \mathrm{kgs}$. BMI of each subject was calculated using Quetlets index.

\section{BMI = Weight (in kg)/Height ${ }^{2}$ (in m)}

Subjects of each gender were divided into three groups based on their BMI. Group 1 comprised of those whose BMI was $<18.5 \mathrm{~kg} / \mathrm{m}^{2}$. Group 2 of individuals with BMI ranging between 18.5-24.99 $\mathrm{kg} / \mathrm{m}^{2}$ and group 3 of those with BMI $>25 \mathrm{~kg} / \mathrm{m}^{2}$.

\subsection{Recording of Auditory Reaction Time and Visual Reaction Time}

The auditory reaction time and visual reaction time were measured by using reaction time apparatus. This apparatus is a portable research reaction timer with 2 response choices latest manufactured in March 2004 and was purchased from Anand agencies, Pune-2, India.

The apparatus has an inbuilt chronoscope-4 digit chronoscope with least count of 1/1000 seconds.

The apparatus has two sides, one "S"s (Subject) and the other "E"s (Examiner). There are 4 keys, on "E"s side. When the key marked "green light" is pressed on the "E"s, side "S" will see the same on his side.
Similarly for the red light, buzzer and click. The stimulus key is double key. It gives the stimulus and also starts the chronoscope. As soon as the stimulus is received $\mathrm{S}$ lifts his finger from the right or left key. This stops the chronoscope and thus reaction time to any of the stimuli is obtained.

All the subjects are thoroughly acquainted with apparatus and 3 readings were taken after practice trials for 4 stimuli, i.e., Red and Green color for visual reaction time, Tone and Click for Auditory reaction time. Reaction time was recorded using the dominant hand.

\subsection{Statistical Analysis}

Mean was calculated for different parameters. The recorded values were expressed as Mean \pm Standard Deviation. Statistical analysis of the data was done using one-way ANOVA (analysis of variance) which is a group of statistical techniques used to compare the means of two or more samples to see whether they come from same population. This technique expands on the tests for two means, such as the $t$-test. Statistical significance of difference was determined. The $P$ value gives the probability of any observed difference having happened by chance. $P$ value below 0.05 was considered to be statistically significant.

\section{Results}

Table 1 shows the reaction times in different groups of females depicting that the visual reaction time for both the stimuli are longer in underweight and overweight females when compared to those females with normal BMI. Similar findings were also observed for both the auditory stimuli.

The same kind of pattern is also observed in the three groups of males wherein underweight and overweight male subjects show longer reaction times when compared to the normal weight males.

On comparing the reaction time after grouping all the 122 subjects in three groups based on their BMI, it was observed that individuals on either side of the normal 
Table 1 BMI and reaction times in females.

\begin{tabular}{llllll}
\hline \multirow{2}{*}{ BMI (in kg/m ${ }^{2}$ ) } & \multirow{2}{*}{ Number of subjects } & \multicolumn{2}{l}{ Visual reaction time(in msec) } & \multicolumn{2}{l}{ Auditory reaction time (in msec) } \\
\cline { 3 - 6 } & & Green light & Red light & Tone & Click \\
\hline$<18.5$ & 11 & $0.142 \pm 0.01$ & $0.144 \pm 0.13$ & $0.142 \pm 0.15$ & $0.147 \pm 0.01$ \\
$18.5-24.99$ & 32 & $0.14 \pm 0.01$ & $0.136 \pm 0.01$ & $0.137 \pm 0.09$ & $0.143 \pm 0.13$ \\
$>25$ & 11 & $0.15 \pm 0.01$ & $0.15 \pm 0.01$ & $0.146 \pm 0.09$ & $0.152 \pm 0.01$ \\
\hline
\end{tabular}

\section{BMI and reaction times in females}

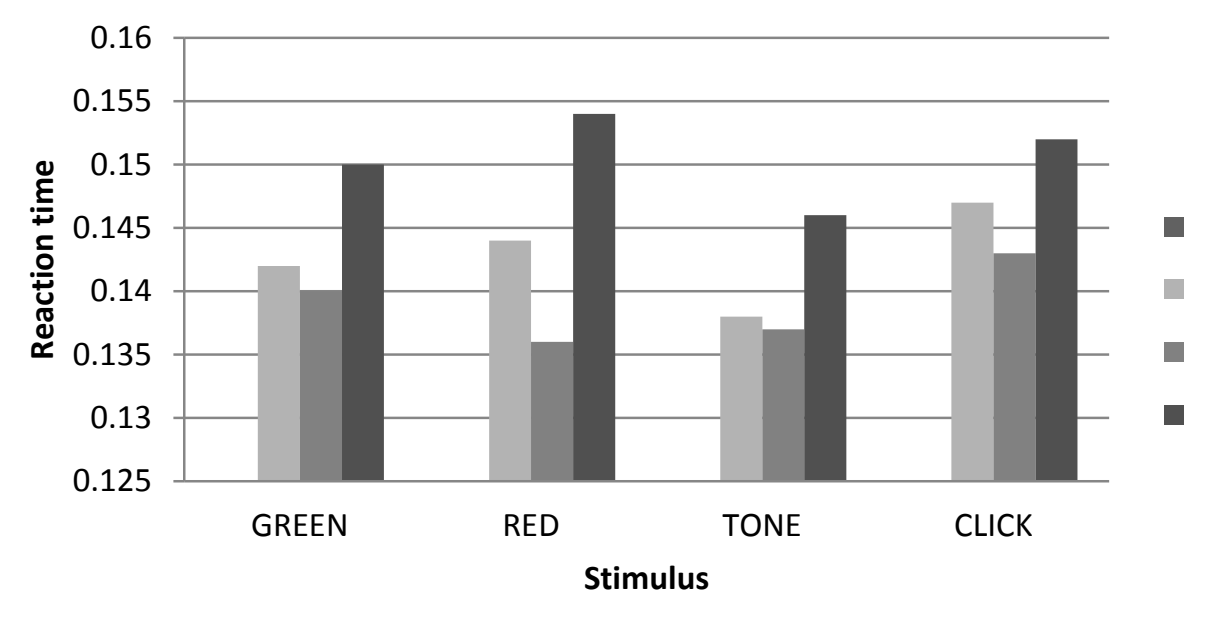

Table 2 BMI and reaction times in males.

\begin{tabular}{llllll}
\hline \multirow{2}{*}{ BMI (in kg/m $\left.{ }^{2}\right)$} & \multirow{2}{*}{ Number of subjects } & \multicolumn{2}{l}{ Visual reaction time(in msec) } & \multicolumn{2}{l}{ Auditory reaction time (in msec) } \\
\cline { 3 - 6 } & & Green light & Red light & Tone & Click \\
\hline$<18.5$ & 13 & $0.146 \pm 0.01$ & $0.145 \pm 0.14$ & $0.138 \pm 0.01$ & $0.144 \pm 0.01$ \\
$18.5-24.99$ & 41 & $0.144 \pm 0.01$ & $0.139 \pm 0.14$ & $0.137 \pm 0.01$ & $0.142 \pm 0.01$ \\
$>25$ & 14 & $0.145 \pm 0.15$ & $0.146 \pm 0.01$ & $0.143 \pm 0.14$ & $0.144 \pm 0.01$ \\
\hline
\end{tabular}

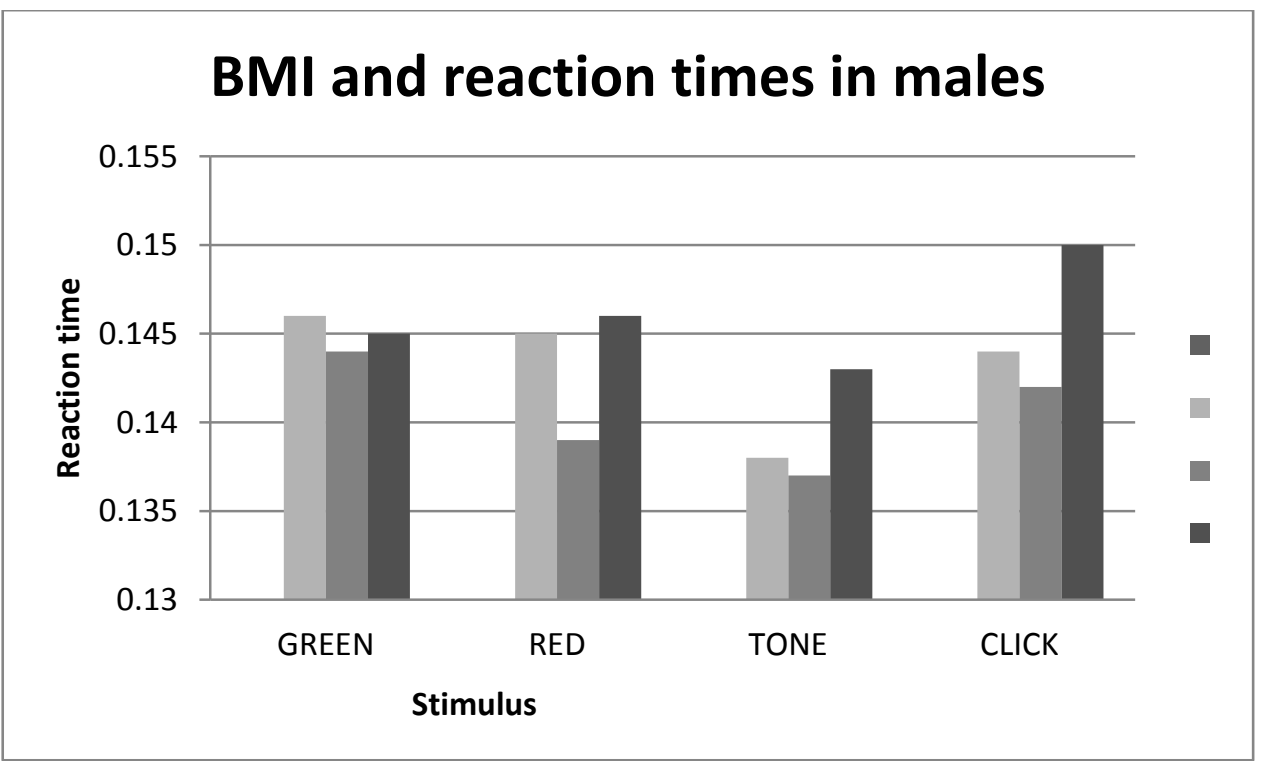


Table 3 BMI and reaction time.

\begin{tabular}{llllll}
\hline \multirow{2}{*}{ BMI (in kg/m ${ }^{2}$ ) } & \multirow{2}{*}{ Number of subjects } & \multicolumn{2}{l}{ Visual reaction time(in msec) } & \multicolumn{2}{l}{ Auditory reaction time (in msec) } \\
\cline { 3 - 6 } & & Green light & Red light & Tone & Click \\
\hline$<18.5$ & 24 & $0.146 \pm 0.01$ & $0.144 \pm 0.01$ & $0.14 \pm 0.01$ & $0.144 \pm 0.01$ \\
$18.5-24.99$ & 73 & $0.143 \pm 0.01$ & $0.147 \pm 0.01$ & $0.137 \pm 0.01$ & $0.142 \pm 0.01$ \\
$>25$ & 25 & $0.148 \pm 0.01$ & $0.138 \pm 0.01$ & $0.144 \pm 0.01$ & $0.15 \pm 0.01$ \\
\hline
\end{tabular}

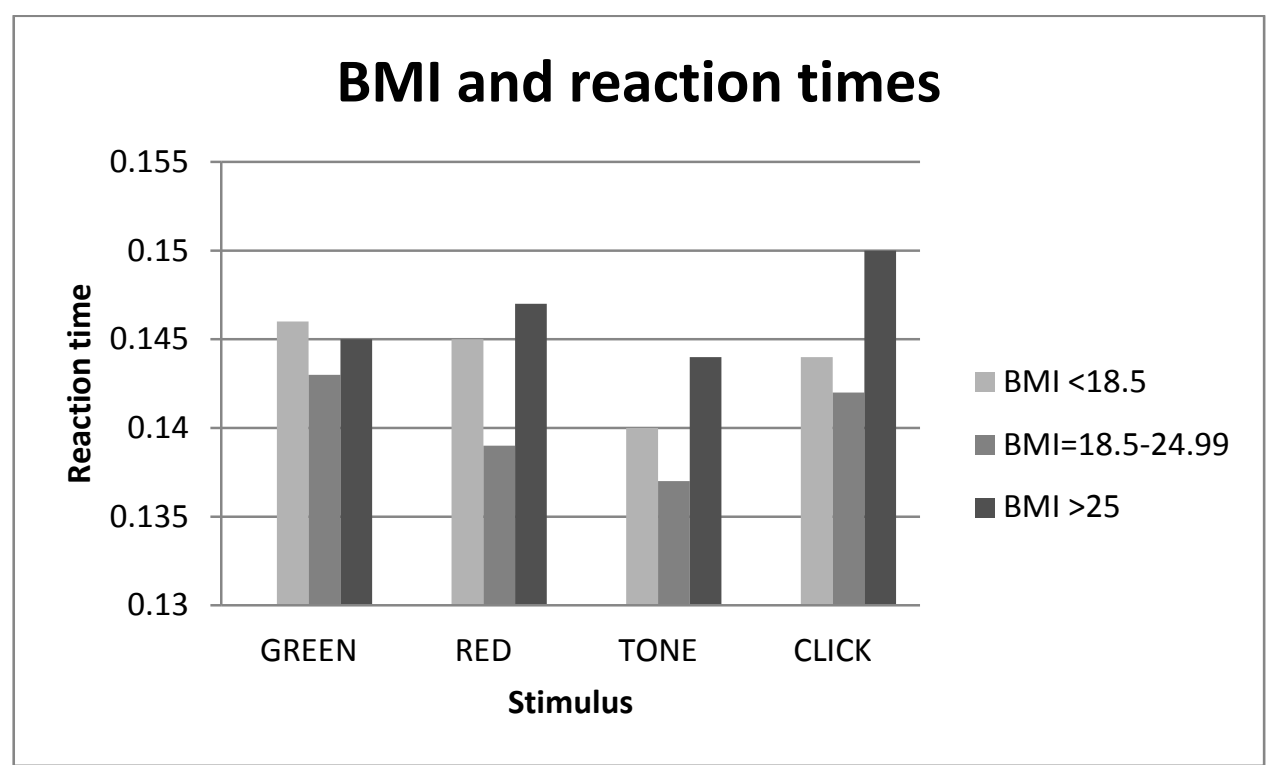

Table 4 Gender and reaction time.

\begin{tabular}{lllll}
\hline Reaction time (in msec) & Stimulus & $\begin{array}{l}\text { Males } \\
(n=68)\end{array}$ & $\begin{array}{l}\text { Females } \\
(n=54)\end{array}$ & ANOVA \\
\hline Visual reaction time & Green light & $0.144 \pm 0.01$ & $0.146 \pm 0.01$ & $\mathrm{~F}=0.52, P>0.05$ \\
& Red light & $0.142 \pm 0.01$ & $0.147 \pm 0.05$ & $\mathrm{~F}=61.18, P<0.001$ \\
\hline \multirow{2}{*}{ Auditory reaction time } & Tone & $0.139 \pm 0.16$ & $0.142 \pm 0.14$ & $\mathrm{~F}=1.27, P>0.05$ \\
& Click & $0.144 \pm 0.14$ & $0.147 \pm 0.14$ & $\mathrm{~F}=1.24, P>0.05$ \\
\hline
\end{tabular}

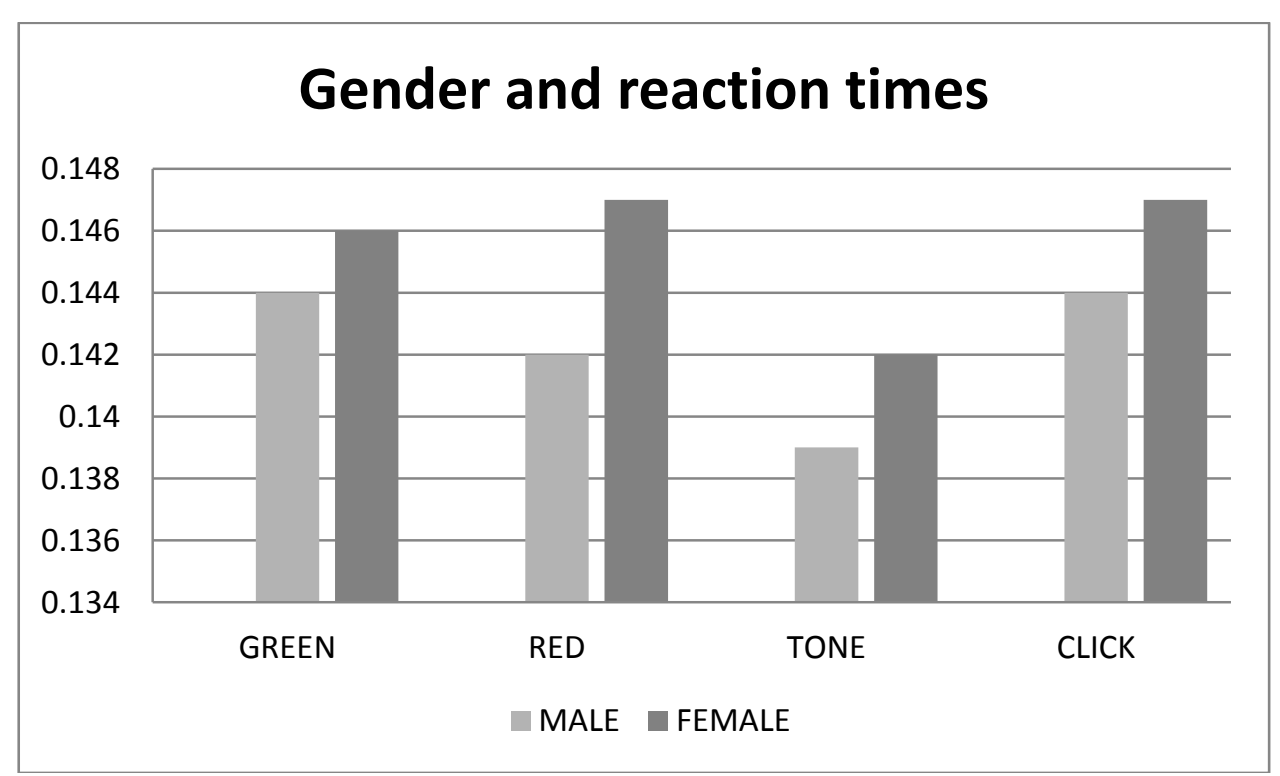


reference range of BMI, i.e., group 1 and group 3 had longer reaction times than those belonging to the group 2.

Table 4 illustrates the reaction time in males and females. It is seen that for all stimuli given, males reacted faster than females. The difference between the reaction time for red visual stimuli is significantly greater for females when compared to males.

\section{Discussion}

The results of our study show that underweight individuals have longer reaction times than individuals of normal weight. This is seen both in males and females as well as when we are comparing the reaction times with BMI irrespective of gender. This can be attributed to cumulative effect of underweight over time on cognition. Underweight could be a result of poor health. A further possibility is that underweight persons experience a dysregulation in hormone secretion corresponding to that in anorexia, which results in cognitive disorders [3]. Further investigation of the mechanisms underlying the cumulative effects of underweight on later cognition would be an important topic for future research.

Also shown in our results is that overweight individuals of either sex react slower than those individuals having normal weight within the same gender group. Irrespective of gender, comparing the reaction time of overweight individuals with normal weight individual's, similar findings were observed. Possible explanation for this could be obesity induced vascular disease. Other mechanisms suggested are secretions of adipose tissue like hormones, cytokines, and growth factors affecting brain health [3]. Different neurophysiological studies have shown influence of obesity and elevated body mass index on cognitive functions, memory deficits and executive dysfunction in young as well as middle aged individuals $[4,5]$.

Our study shows that on comparing the visual reaction time between males and females it is observed that females have longer visual reaction times than males. Females take a longer time to react to red light when compared to males and the difference in the time taken to react has been found to be statistically significant. Similarly, the auditory reaction time in females has been found to be more than those of males, this result although being not statistically significant. This may be due to the retention of water and sodium due to variation in sex steroid levels during menstrual cycles which might influence the process of axonal conduction time and availability of neurotransmitter at the synaptic level; changes in either of these two processes might cause conduction time to vary during menstrual cycle. Studies have shown that the degree of premenstrual weight gain is correlated with degree of various premenstrual neurological symptomatologies [6].

\section{Conclusion}

It is seen that those individuals with a BMI lower than the normal range, have longer reaction times than those individuals that belong to the normal range of BMI. Same finding also holds true for those individuals that have a higher than normal BMI. This shows the importance of being in the normal BMI range. Therefore, the need for a healthy lifestyle, good eating habits and regular exercise undoubtedly requires emphasis all the more.

Also observed is that females react slower to both visual and auditory stimuli, but further research in this field with a larger sample size will be needed to clear the debate as to whether gender should be taken as a criteria for the appointment of personnel in certain fields.

\section{Drawback}

The menstrual phase of each female subject was not recorded and hence there was no uniformity based on that criteria for the selection of female subjects as there are studies also depicting the effect of different phases of menstrual cycle on reaction time. 


\section{References}

[1] Gandhi, P. H., Gokhale, P., Mehta, H. B., and Shah, C. J. 2013. "A Comparative Study of Simple Auditory Reaction Time in Blind (Congenitally) and Sighted Subjects." Indian J. Psychol. Med. 35 (3): 273-7.

[2] Nikam, L. H., and Gadkari, J. V. 2012. "Effect of Age, Gender and Body Mass Index on Visual and Auditory Reaction Times in Indian Population." Indian J. Physiol. Pharmacol. 56 (1): 94-9.

[3] Nene, A. S., Pazare, P. A., and Sharma, K. D. 2011. "A Study of Relation between Body Mass Index and Simple
Reaction Time in Healthy Young Females." Indian $J$. Physiol. Pharmacol. 55 (3): 288-91.

[4] Cournot, M., Marquie, J. C., and Ansiau, D. 2006. "Relation between Body Mass Index and Cognitive Function in Healthy Middle Aged Men and Women." Neurology 67 (7): 1208-14.

[5] Gunstad, J., Paul, R. H., Cohaen, R. A., Tate, D. F., and Gordon, E. 2006. "Obesity Is Associated with Memory Deficits in Young and Middle Aged Adults." Eat Weight Disord. 11: 15-9.

[6] Bamne, S. N., and Ameet, D. 2011. "Effect of Color and Gender on Human Reaction Time." Indian J. Physiol. Pharmacol. 55 (4): 388-9. 